\title{
Full-field birefringence imaging by thermal-light polarization-sensitive optical coherence tomography. II. Instrument and results
}

\author{
Julien Moreau, Vincent Loriette, and Albert-Claude Boccara
}

\begin{abstract}
We describe an instrument for measuring the magnitude of birefringence of tomographic images and the principal directions of axes that uses thermal-light polarization-sensitive optical coherence tomography. The instrument permits full-field measurements with an axial resolution of $1.5 \mu \mathrm{m}$ and a transverse resolution limited by diffraction. We obtained a sensitivity of $84 \mathrm{~dB}$, limited by shot noise, when we integrated the signal for $1 \mathrm{~s}$. We verified the validity of the measurement by measuring the birefringence of a variable phase shifter. We present typical results obtained with optical samples. (C) 2003 Optical Society of America
\end{abstract}

OCIS codes: $110.4500,120.5060,260.1440$.

\section{Introduction}

Optical coherence tomography (OCT) is an attractive technique for obtaining information about buried structures with high spatial resolution. OCT is oriented mainly toward the study of diffusive samples, but it can be used as well for testing transparent samples with low optical contrast. In this case polarization-sensitive OCT, which brings the advantages of dark-field microscopy, proves to be an effective technique for revealing structures that otherwise would hardly be detectable. ${ }^{1-9}$ The combination of a polarization-sensitive instrument with a broadband source allows one to obtain polarizationdependent information with micrometer-scale resolution in three dimensions. With a CCD array, full-field microscopy is possible. ${ }^{10-12}$ The coherence length of the broadband source determines the axial resolution of the instrument, so microscope objective lenses with large depths of field or low numerical apertures can be used without spoiling the axial resolution. Unless one uses immersion

The authors are with the École Supérieure de Physique et Chimie Industrielles de la Ville de Paris, Laboratoire d'Optique Physique, Centre National de la Recherche Scientifique Unité Propre de Recherche 5, 10 rue Vauquelin, 75005 Paris, France. V. Loriette's e-mail address is loriette@optique.espci.fr.

Received 13 September 2002; revised manuscript received 28 January 2003.

0003-6935/03/193811-08\$15.00/0

(C) 2003 Optical Society of America objectives and samples with low index variations, the use of low-numerical-aperture objectives is obligatory when one tests samples that exhibit large index changes because aberrations inside the sample put severe limitations on the depth that can be probed. However, the use of a broadband source raises a major technical problem: Although achromatic polarizers can be manufactured easily, making a fairly achromatic phase-shifting component is a difficult task. The most common achromatic phase-shifting component is the Fresnel rhomb. Standard commercially available Fresnel rhombs exhibit phase errors of a few degrees over a span of a few hundred nanometers. Unfortunately the standard design of Fresnel rhombs makes them unsuitable for OCT microscopy because they shift the emerging beam laterally and are highly sensitive to misalignment. The use of multilayer phaseshifting plates permits the problem of beam shift to be overcome, but phase-shifting plates are far less efficient than rhombs in the blue part of the spectrum, and their performance varies greatly from one manufacturer to the other. The present authors have chosen to use achromatic quarter-wave plates, chiefly because of their ease of use, although, for some experimental configurations, this choice is made to the detriment of accuracy and sensitivity. In Section 2 of this paper we describe the experimental arrangement, and in Section 3 we discuss its performance. We focus on elimination of the spurious signal induced by phase errors in the quarter- 


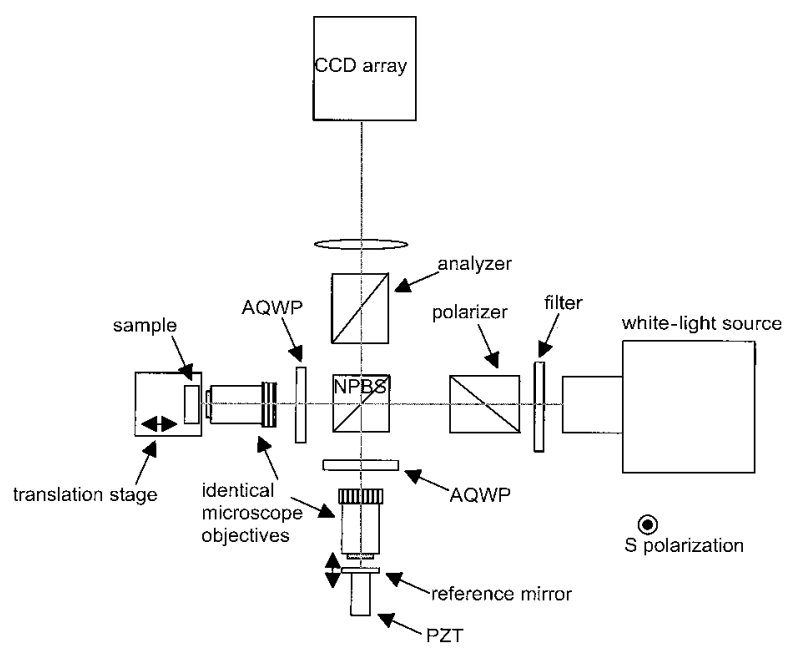

Fig. 1. Schematic of the instrument described in this paper: NPBS, nonpolarizing beam-splitter cube; AQWPs, achromatic quarter-wave plates; PZT, piezoactuated translation stage.

wave plates. In Section 4 we present some results for multilayer optical samples.

\section{Experimental Arrangement}

The experimental arrangement is sketched in Fig. 1. The instrument is based on a Linnik interference microscope, i.e., a Michelson interferometer with a microscope objective lens in each arm. The reference surface should have topographic features much smaller than the sample, and to optimize the contrast its reflectance should be as close as possible to the reflectance of the sample. Uncoated glass, which has a low reflection factor of $\sim 4 \%$ and can be superpolished, is a good choice when one is testing biological or transparent optical samples. Light is emitted by a xenon lamp with $300-\mathrm{nm}$ bandwidth centered about $800 \mathrm{~nm}$. It is polarized with a Glan-Taylor polarizer that has an extinction ratio of $2 \times 10^{-5}$. The beam is divided by a nonpolarizing beam-splitter cube. Each arm contains an achromatic quarter-wave plate (Newport Corporation Model ACWP) and a $20 \times$, microscope objective with a N.A. of 0.4 (Olympus $20 \times$ plan achromat). The emerging light is collected by a CCD array $(256 \times 256$ pixel, 8-bit, maximum $200-\mathrm{Hz}$ readout frequency; Dalsa Semiconductors Model CAD-1) after it has passed through a thin plate polarizer with an extinction ratio of $4 \times 10^{-5}$. The plate polarizer is fixed upon a motorized rotation stage to permit angular positioning with a precision of $0.17 \mathrm{mrad}$. Both reference mirror and sample are fixed upon motorized translation stages. The reference is attached to a piezoelectric actuator oscillating at $45 \mathrm{~Hz}$, and the sample is translated with a 74-nm step motorized translation stage. The amplitude of piezo-oscillation can be adjusted to produce a particular modulation depth. The piezoactuator driver and the CCD are phase locked by two waveform generators (Hewlett-Packard Model 33120A). The total acquisition time depends on

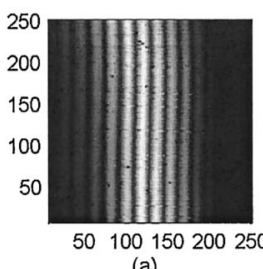

(a)

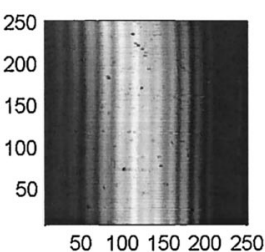

(b)

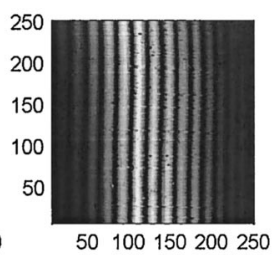

(c)
Fig. 2. Procedure used to fix the value of $\delta_{0}$ : interferograms with (a) $\delta_{0}=0.85 \times \delta_{\text {oopt }}$, (b) $\delta_{0}=\delta_{0 \text { opt }}$, and (c) $\delta_{0}=1.15 \times \delta_{\text {oopt }}$.

the number of averages for each position of the sample and on the total volume scanned; a good order of magnitude is $4 \mathrm{~s} /$ section for a 100-image average.

\section{Setup and Measurement Procedure}

The orientations of the various polarization components are adjusted by use of extinction configurations. At the end of this step the polarizer is oriented to produce a $P$-polarized field incident upon the beam splitter. The quarter-wave plate in the sample arm is oriented such that its fast axes make an angle of $45^{\circ}$ with the $P$ axis, and the quarter-wave plate in the reference arm is oriented at $11^{\circ}$. These values allows the signal-to-noise ratio to be maximized, as explained below. During this step of the setup the CCD is replaced by a silicon photodetector for better sensitivity. With digital waveform generators, both modulation depth and phase are adjusted independently by use of the method discussed in Ref. 13. By measuring four images with a tilted nonbirefringent test sample and calculating $S_{2}{ }^{(P)}-S_{4}{ }^{(P)}$ we can adjust the modulation phase to $\pi / 4$. By making the fringes disappear at the center of the interferogram, as shown in Fig. 2, we can fix the modulation depth close to its theoretical value of 2.0759 rad with an estimated precision of $98 \%$. Once the modulation parameters are fixed, the nonbirefringent test sample is replaced by the sample that we wish to study. We then record two sets of four images $\left\{S_{i}^{(S)}, S_{i}^{(P)}\right\}$, $i=1-4$, acquired with two different orientations, $S$ and $P$, of the analyzer. For a given analyzer position the corresponding four images are acquired consecutively; each image is integrated during one quarter of the modulation period. This eightimage acquisition is performed for each position of the sample. We form four linear combinations:

$$
\begin{aligned}
& \Sigma_{B}{ }^{(S)}=-S_{1}{ }^{(S)}+S_{2}{ }^{(S)}+S_{3}{ }^{(S)}-S_{4}{ }^{(S)}, \\
& \Sigma_{A}{ }^{(S)}=-S_{1}{ }^{(S)}+S_{2}{ }^{(S)}-S_{3}{ }^{(S)}+S_{4}{ }^{(S)}, \\
& \Sigma_{B}{ }^{(P)}=-S_{1}{ }^{(P)}+S_{2}{ }^{(P)}+S_{3}{ }^{(P)}-S_{4}{ }^{(P)}, \\
& \Sigma_{A}{ }^{(P)}=-S_{1}{ }^{(P)}+S_{2}{ }^{(P)}-S_{3}{ }^{(P)}+S_{4}{ }^{(P)} ;
\end{aligned}
$$




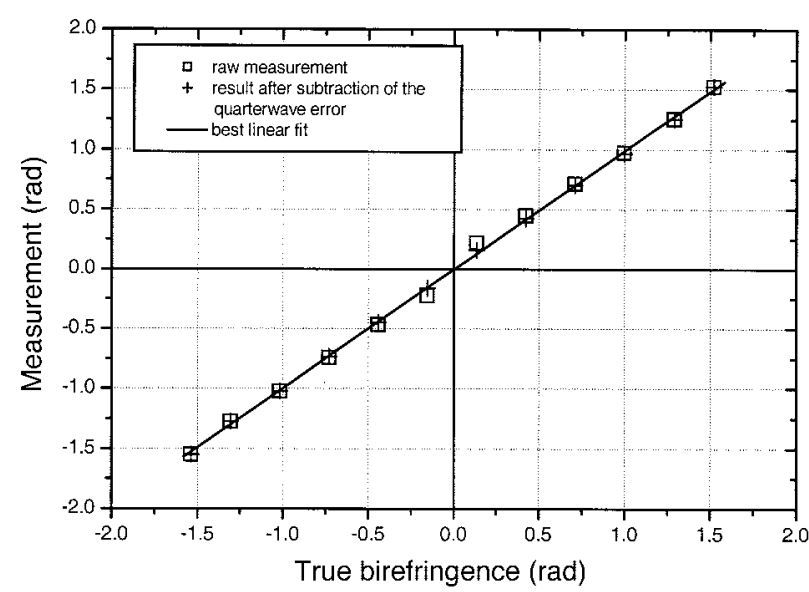

Fig. 3. Relation of estimated to true retardation of a Babinet compensator: squares, uncorrected data; crosses, data corrected for quarter-wave plate error following to Eq. (15).

and calculate birefringence magnitude $\delta_{B}$, orientation $\beta$, and signal topography $\delta_{z}$ with the formulas

$$
\begin{aligned}
\tan ^{2}\left(\pi \frac{\delta_{B}}{\lambda_{0}}\right) & =\tan ^{-2}(2 \theta) \frac{\left[\Theta_{B} \Sigma_{A}{ }^{(P)}\right]^{2}+\left[\Theta_{A} \Sigma_{B}{ }^{(P)}\right]^{2}}{\left[\Theta_{B} \Sigma_{A}{ }^{(S)}\right]^{2}+\left[\Theta_{A} \Sigma_{B}{ }^{(S)}\right]^{2}}, \\
\tan \left(2 \pi \frac{\delta_{z}}{\lambda_{0}}\right) & =\frac{\Theta_{B} \Sigma_{A}{ }^{(S)}}{\Theta_{A} \Sigma_{B}{ }_{B}^{(S)}} \\
\tan (2 \beta) & =\frac{\Theta_{A} \Theta_{B}\left[\Sigma_{A}{ }^{(S)} \Sigma_{B}{ }^{(P)}-\Sigma_{A}{ }^{(P)} \Sigma_{B}{ }_{B}^{(S)}\right]}{\Theta_{A}{ }^{2} \Sigma_{B}{ }^{(S)} \Sigma_{B}{ }^{(P)}+\Theta_{B}{ }^{2} \Sigma_{A}{ }^{(S)} \Sigma_{A}{ }^{(P)}},
\end{aligned}
$$

where, knowing the apparent spectrum of the source, we calculate $\Theta_{A}$ and $\Theta_{B}$, and $\lambda_{0}$ is an equivalent wavelength of the polychromatic source.

\section{Results}

We calibrated our instrument by inserting a Babinet compensator into the sample arm between the quarter-wave plate and the microscope objective. We generated a well-defined birefringence signal with known axial directions and varied its amplitude from $-\pi / 2$ to $+\pi / 2$. Figure 3 shows the measured birefringence as a function of the true retardation imposed by the compensator. The best linear fit is shown (solid line); its coefficient of linear regression is 0.99985 , slope is $0.993 \pm 0.005$, and intercept is $-3 \pm$ $5 \mathrm{mrad}$, demonstrating the good agreement of the measurement with the true values. However, it must be noted that to obtain this result we found it necessary to take into account the imperfections of the quarter-wave plates. This correction is described in Section 5 below.

We validated our setup with a multilayer optical coating sample whose structure is sketched in Fig. 4. For an optical system with a scattering specification level of a few parts in $10^{6}$ (laser gyros or interferometric detectors of gravitational waves ${ }^{14,15}$ ), point defects inside the multilayer coating are a major source of loss. Knowing the locations of these scattering

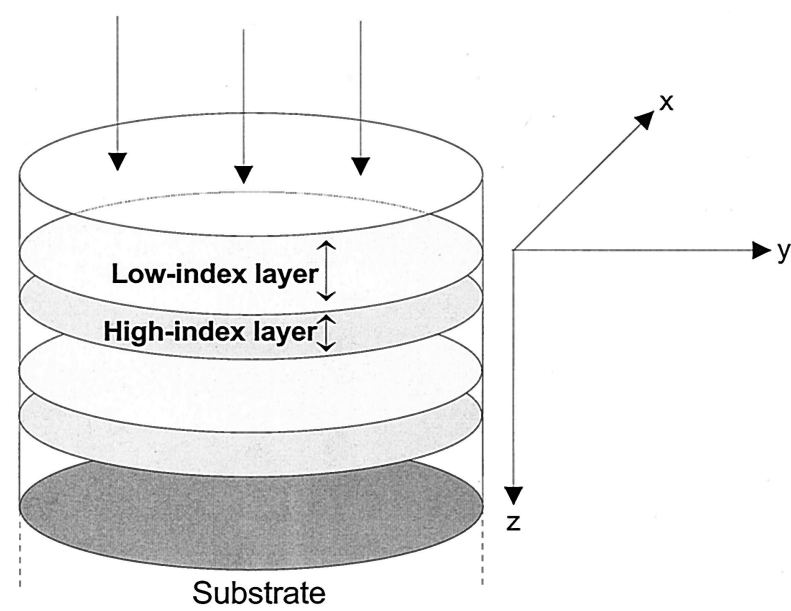

Fig. 4. Schematic of the multilayer optical coating mirror used as a sample.

defects, and furthermore using a nondestructive technique, will help in finding the source of contamination. This detection cannot be made by use of a tomographic image of the reflectance of the structure because the specular light from the interfaces would mask the small signal coming from the defect. However, if the scattering defect is nonspherical, it can change the polarization of the incident light. Therefore, in a tomographic image of the birefringence the point defect will appear as a bright spot in a dark field, as the interfaces are not birefringent. Our sample is an infrared interference coating designed for a $\mathrm{CO}_{2}$ laser $(\lambda=10.6 \mu \mathrm{m})$, with five layers of alternate high- and low-index dielectric material deposited upon a ZnSe substrate. The layers are alternately half-wave and quarter-wave plates. Figure 5 shows the interferogram envelope: $\Omega(z),{ }^{13}$ which in this case is similar to the classic OCT to-

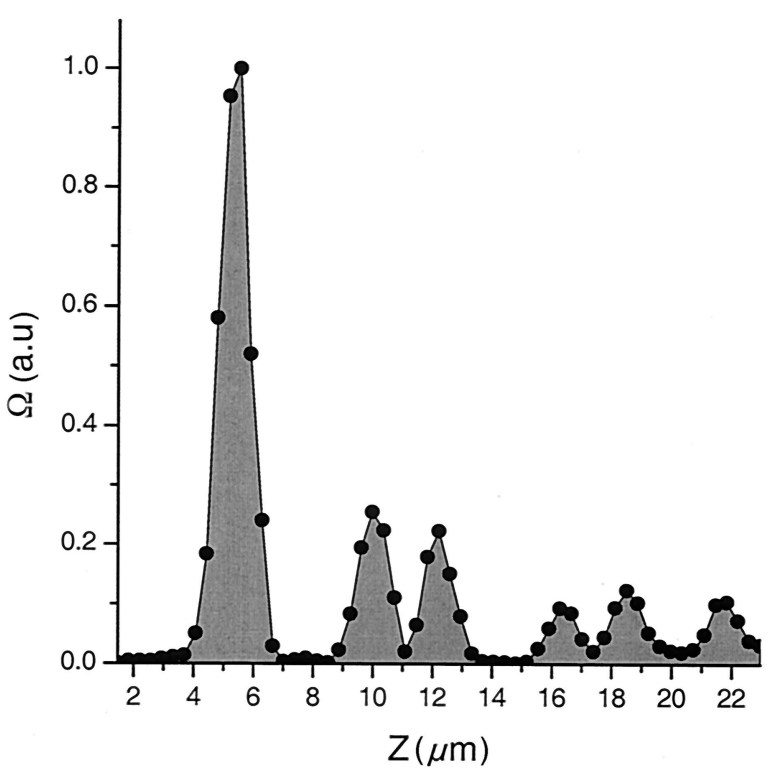

Fig. 5. Classic OCT tomography cut of the multilayer. Six interfaces can be distinguished. 


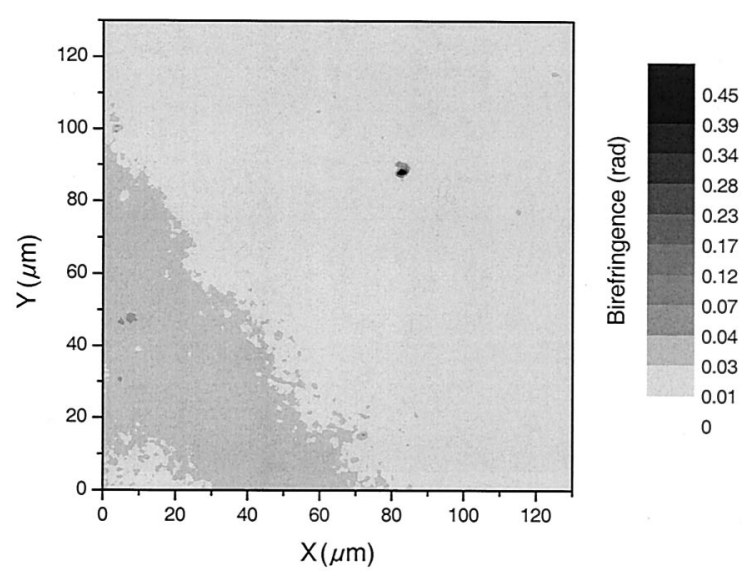

Fig. 6. En face birefringence image of the multilayer second interface on which a defect is visible: $X Y$ cut, $0.7 \mu \mathrm{m} \times 0.7 \mu \mathrm{m}$ resolution. The noise in this image is estimated to be $2.9 \times 10^{-4}$ rad.

mography signal. It must be noted that the scale of the $Z$ axis is given by the translation of the sample upon its motorized stage. To obtain true optical thickness values one should correct the $Z$ scaling by an obliquity factor that depends on the objective's N.A. and on the sample's index of refraction. The axial resolution of our instrument enables us to resolve the six interfaces. We made a threedimensional birefringence map of this mirror $(60$ images; step, $0.4 \mu \mathrm{m}$ ) to enable us to detect the presence of point defects inside the structure. Figures 6 and 7 , respectively, show $X Y$ cuts of the magnitude and direction of the birefringence axes about such defects. Figures 8 and 9 , respectively, show $X Z$ and $Y Z$ cuts. In these cuts the noise was measured to be

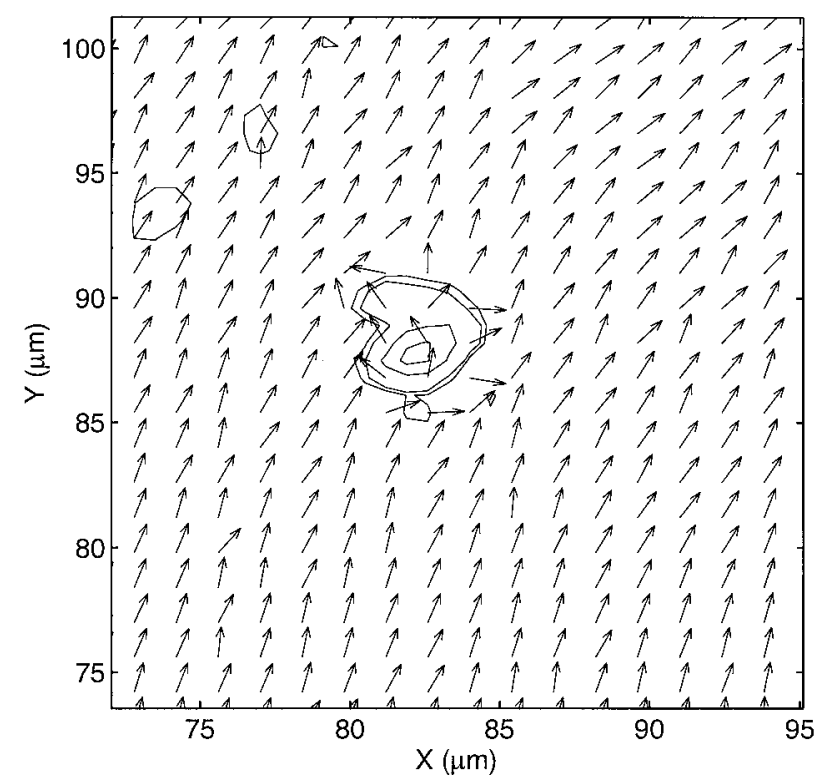

Fig. 7. Direction of the birefringence axes in the region about the defect on the multilayer second interface; $X Y$ cut. The contour lines indicate the locations of the birefringent structures.

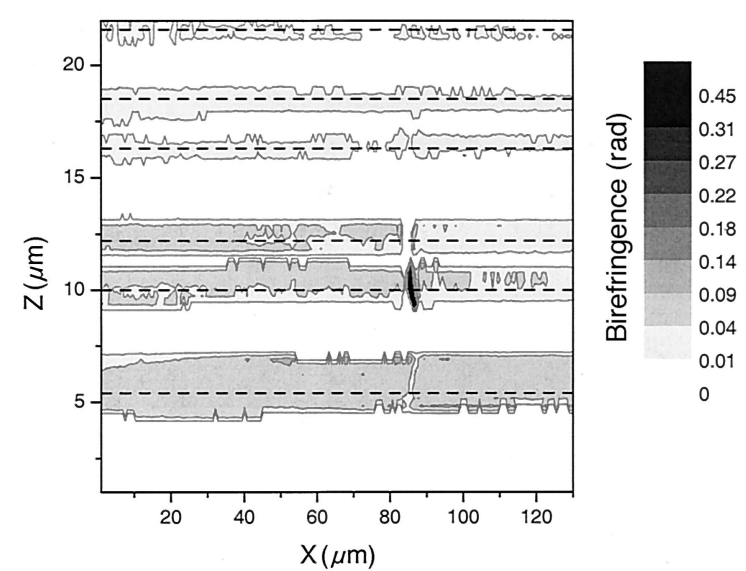

Fig. 8. High-resolution $(0.7 \mu \mathrm{m} \times 1.5 \mu \mathrm{m}$, lateral $\times$ axial $)$ birefringence image of the multilayer; $X Z$ cut; same noise level as in Fig. 6. Dashed lines, positions of the six interfaces.

$2.9 \times 10^{-4} \mathrm{rad}$. Figure 7 shows a measurement of the direction of the birefringence axes in the region where the defect was detected. To ensure quantitative measurement we evaluated the birefringence only when $\Omega$ took a significant value. A defect in birefringence can be seen and precisely located on the second interface. Its size is equal to or less than the resolution of our instrument in three dimensions. The spurious birefringence signal coming from the interfaces $(\sim 0.1 \mathrm{rad})$ is due to a retardation error of the achromatic quarter-wave plate (see Subsection 5.D below). The time needed to acquire a complete three-dimensional map of a sample depends on the number of $X Y$ cuts as well as on the number of images averaged at each position of the sample. In the measurement presented, the sample was moved to 60 different $Z$ positions, and an average of 400 sequences was performed at each position, so the total number of images acquired was $60 \times 8 \times 400$. The time needed for the sample to move from one position to the next was $200 \mathrm{~ms}$, and the total acquisition time was $17 \mathrm{~min}$.

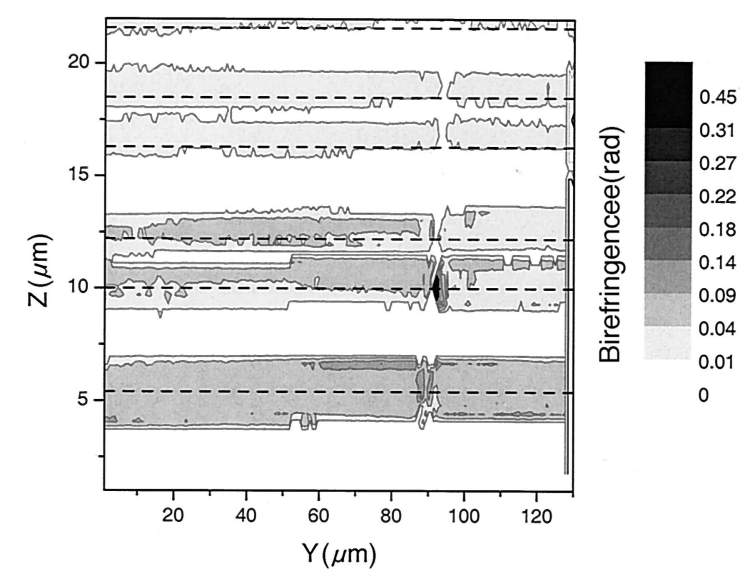

Fig. 9. $Y Z$ cut. 


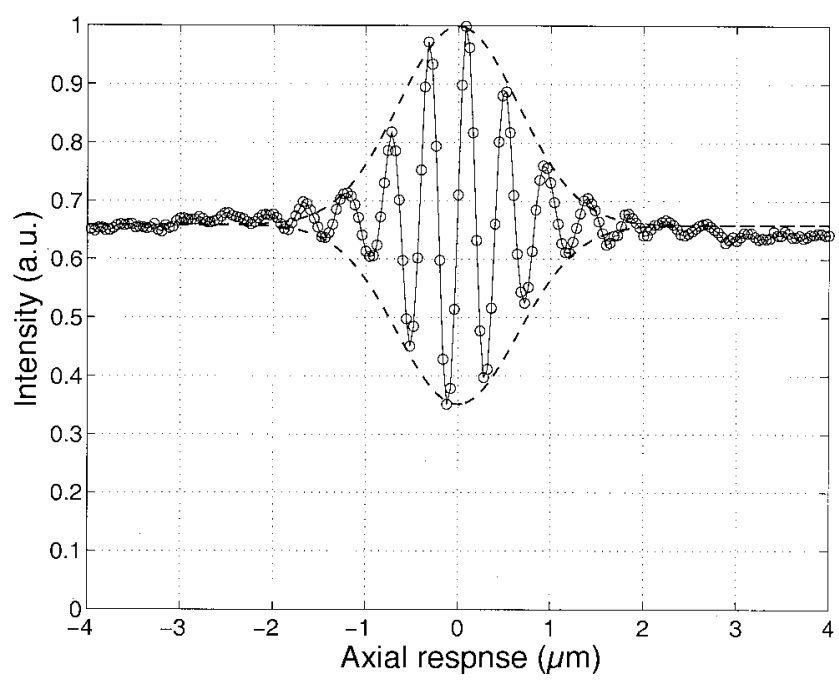

Fig. 10. Measured axial response of the system and best Gaussian envelope. The FWHM is $1.5 \mu \mathrm{m}$.

\section{Performance and Limitations}

\section{A. Resolution and Position Accuracy}

The axial resolution in air is evaluated with a single interferogram recorded by use of a mirror sample. From the measurement presented in Fig. 10 one can find the best Gaussian envelope of the interferogram and estimate the full-width at half-maximum. We found axial resolution $\Delta_{z}=1.5 \mu \mathrm{m}$ in air. The axial resolution is limited by the apparent spectrum width of the light source presented in Fig. 11 (i.e., which takes into account the spectral response of the CCD array and of the optical components) and by uncompensated dispersion inside the interferometer and inside the sample. The uncompensated dispersion can

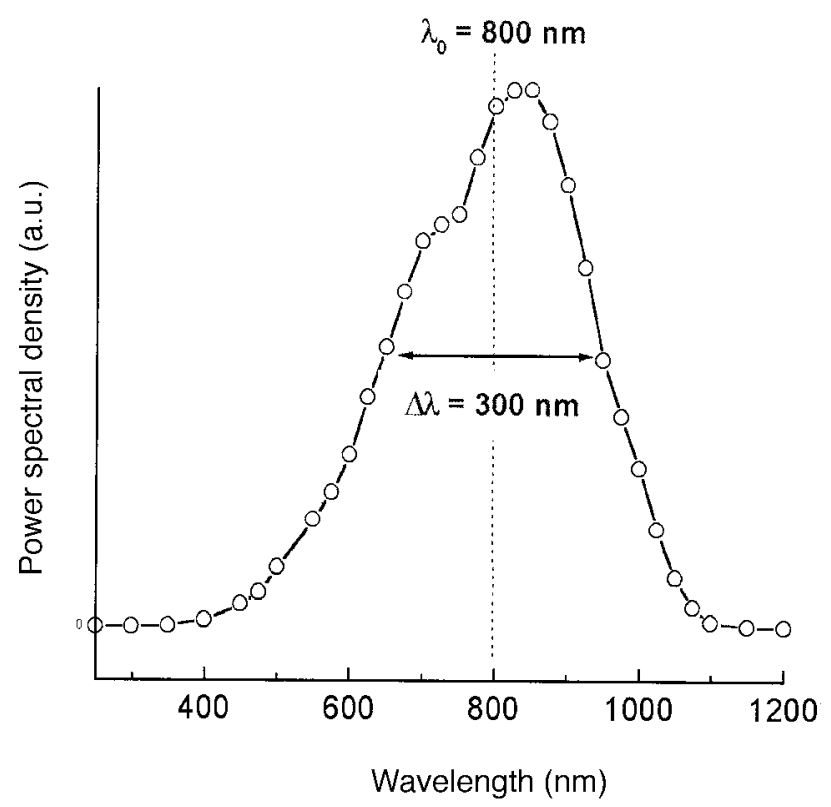

Fig. 11. Spectrum of the light source used in the experiment as a function of wavelength. in principle be corrected if one knows the dispersion curves and the thicknesses of the various optical elements and sample. The accuracy of the axial position depends on the algorithm that uses one to find the position of the maximum of the interferogram envelope and is limited by the axial step size of the sample displacement. In our setup the displacement step size is $74 \mathrm{~nm}$, which can be taken as a good order of magnitude of the accuracy of the axial position.

The lateral resolution in air is limited by diffraction and depends on the objective lenses that are used. We use an optical scheme in which each pixel of the CCD array receives the image of a cross section of the sample approximately equal to the diffraction spot size, so the accuracy of the lateral position is equal to the resolution. When better lateral accuracy is required, expanding imaging optics can be added such that the diffraction spot is imaged on more than a single pixel.

\section{B. Maximum Detectable Birefringence}

The short coherence length limits the maximum magnitude of birefringence that can be detected: If the retardation induced by the sample is larger than the coherence length of the source, then, after interaction with the sample, the two eigenstates of polarization will lose their mutual coherence and the field returning from the reference arm will become a superposition of two orthogonally polarized incoherent fields. The maximum value of the birefringence that can be measured is $\max \left(\delta_{B}\right)=\Delta_{z}$, or, expressed in terms of phase retardation and using the equivalent wavelength of the source,

$$
\max (\varphi)=2 \pi \frac{\Delta_{z}}{\lambda_{0}} .
$$

In our setup this value is $\sim 3 \pi$.

\section{Sensitivity}

The performance of the instrument is limited by two factors, residual noise and imperfections and misorientation of the polarizing components, especially the quarter-wave plates and the beam-splitter cube. The former puts an upper limit on sensitivity and the latter, together with uncertainties in the modulation parameters, degrade measurement precision. The fundamental sensitivity limits of the instrument are discussed in depth in the paper by Dubois. ${ }^{16} \mathrm{We}$ measured the signal-to-noise ratios (SNRs) of individual images and found that they are shot-noise limited up to an integration duration of $20 \mathrm{~s}$. During 1-s integration we measured a SNR of $70 \mathrm{~dB}$; the theoretical value of the shot-noise-limited SNR for a $13 \times 10^{4}$ charge per pixel CCD is $73 \mathrm{~dB}$. Usually in OCT-related papers the SNR is given as a minimum sample reflectance, measured with a glass reference surface. ${ }^{16}$ In that case a correction equal to $-20 \times$ $\log _{10}\left(\sqrt{R_{\text {glass }}}\right)=14 \mathrm{~dB}$ is added to the SNR value, which in the study reported here increases to $84 \mathrm{~dB}$. When the duration of integration becomes large, 


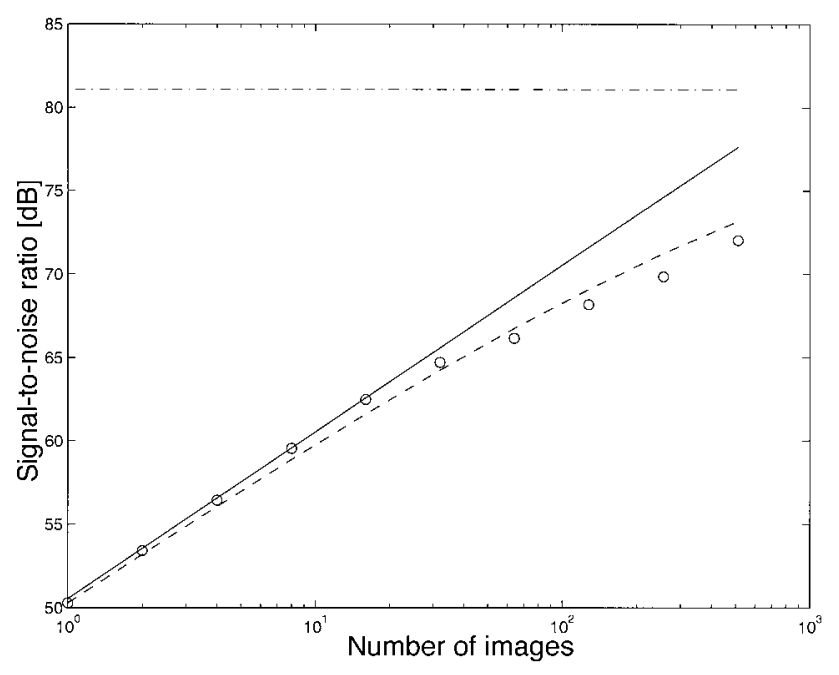

Fig. 12. Image SNR as a function of the number of images averaged. Circles, measurements; solid line, shot-noise limit; dashed curve, estimate of the SNR if an $f^{-1}$ noise source is present; horizontal dashed-dotted line, SNR limit imposed by the $f^{-1}$ noise.

other sources of noise, possibly mechanical, acoustic, and thermal, become dominant.

The shape of the measured sensitivity-versus-time plot presented in Fig. 12 suggests that this technical noise may exhibit an $f^{-1}$ frequency dependency, and its presence places an upper limit of $81 \mathrm{~dB}(95 \mathrm{~dB}$ in terms of minimum reflectance with an uncoated glass reference) on the SNRs of individual images. However, we are interested not in the SNRs of individual images but in the SNR of the birefringence measurement. The way to compute the SNR of the birefringence measurement, $\operatorname{SNR}(\varphi)$, starting from the SNR of individual images, $\operatorname{SNR}(S)$, is straightforward but lengthy, and we simply outline it here. We start our calculation by assuming that each image exhibits the same average value and noise level as the other images and that the noise of the various images is uncorrelated. These assumptions allow to calculate the SNRs of the various combinations of $\Sigma_{A}{ }^{(S)}, \Sigma_{A}{ }^{(P)}$, $\Sigma_{B}{ }^{(S)}$, and $\Sigma_{B}{ }^{(P)}$, using Eqs. (1)-(4), and then the SNR of the birefringence measurement, using Eq. (5). There is a simple relation between the SNRs of individual images $\operatorname{SNR}(S)$ and the SNR of the birefringence measurement $\operatorname{SNR}(\varphi)$ that depends on the magnitude of the birefringence and on the orientation $\theta$ of the quarter-wave plate in the reference arm:

$$
\operatorname{SNR}(\varphi)=\operatorname{SNR}(S) \times g(\varphi, \theta),
$$

where $g$ is a known function of $\varphi, \theta$, and the modulation amplitude. The plot of $g$ in Fig. 13, calculated for $\Theta_{A}=\Theta_{B}$, shows that the value of $\theta$ that gives the highest SNR for the broadest range of $\varphi$ is $\sim 11^{\circ}$ [the optimal value is $22.5^{\circ}$ only when one is interested in measuring $\tan (\varphi / 2)]$. Figure 14 is a plot of $g\left(\varphi, 11^{\circ}\right)$, which shows that $\operatorname{SNR}(\varphi)$ is roughly ten times less than $\operatorname{SNR}(S)$. We thus estimate the maximum SNR for a birefringence measurement to be $61 \mathrm{~dB}(50 \mathrm{~dB}$ for 1-s integration).

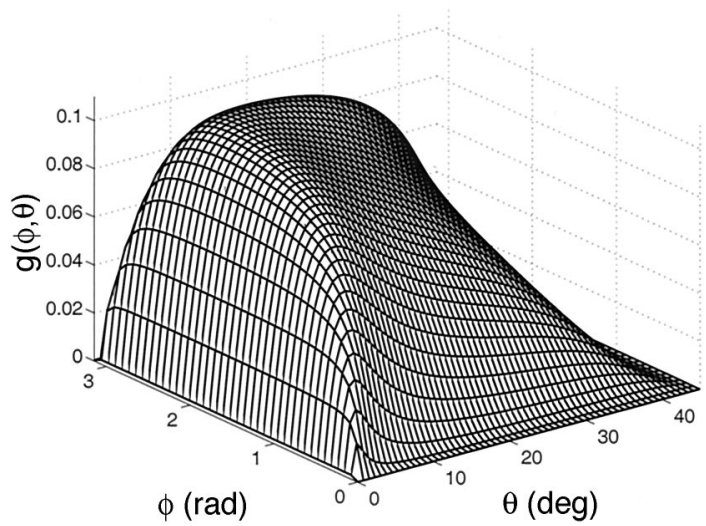

Fig. 13. Value of $g$ as a function of birefringence magnitude $\varphi$ and orientation of the quarter-wave in reference $\operatorname{arm} \theta$.

\section{Precision}

The most important limitation on instrument precision comes from the various imperfections of the optical components, in particular, their behavior with respect to polarization. The requirement of achromaticity is scarcely achievable in polarization components. For polarizers it appears as a poor extinction factor; for phase-shifting components, as spurious wavelength dependent retardation. To eliminate any displacement of the image on the CCD when we rotate the analyzer, we have to use a thin polarizer with an extinction two times larger than one could obtain with a Glan-type polarizer. Then, as was found experimentally, coupling of polarization states can occur inside the beam-splitter cube if the cube is slightly misaligned with respect to the axes of the two arms. It is rather difficult to overcome this problem because it does not show in pure intensity images and is hardly distinguishable $a$ priori for misorientations of the polarizer or the analyzer. The optical components that cause the largest imperfections are the two achromatic quarter-wave plates, as we emphasized above. A simple calculation can be performed for a monochromatic source in the presence of spurious retardation of $\delta_{Q}$ waves in the quarter-wave

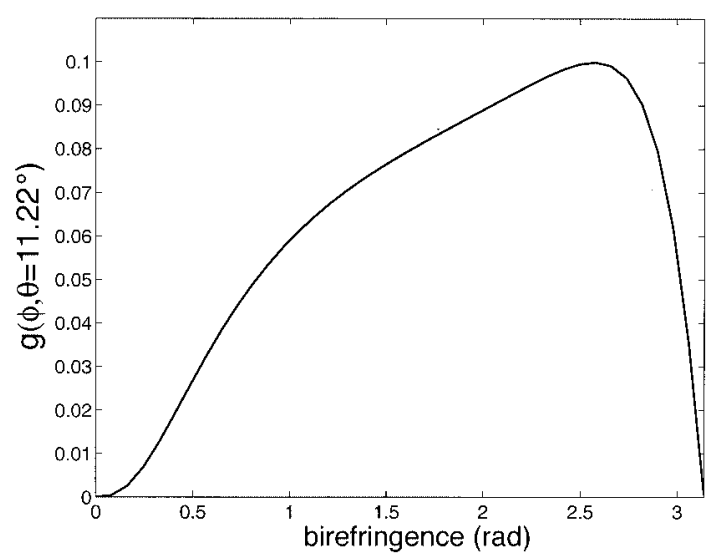

Fig. 14. Value of $g$ as a function of birefringence magnitude for $\theta=11.26^{\circ}$. 
plates. In this case the Jones matrix of the quarterwave plates reads as

$$
M_{Q}=\left[\begin{array}{cc}
\exp \left[i\left(\frac{\pi}{4}+\delta_{Q}\right)\right] & 0 \\
0 & \exp \left[-i\left(\frac{\pi}{4}+\delta_{Q}\right)\right]
\end{array}\right] .
$$

The apparent birefringence of the sample, $\varphi_{\text {app }}$, calculated with the standard equation that is valid for the monochromatic source, is related here to the true value of the birefringence by

$$
\tan ^{2}\left(\frac{\varphi_{\text {app }}}{2}\right)=\frac{\sin ^{2}(\varphi / 2)+h\left(\varphi, \beta, \delta_{Q}\right)}{\cos ^{2}(\varphi / 2)-h\left(\varphi, \beta, \delta_{Q}\right)},
$$

with

$$
\begin{aligned}
h\left(\varphi, \beta, \delta_{Q}\right)= & {\left[\cos ^{2}(\varphi / 2)\right.} \\
& \left.-\sin ^{2}(2 \beta) \sin ^{2}(\varphi / 2)\right] \sin ^{2}\left(2 \delta_{Q}\right) \\
& -\sin (\varphi) \sin (2 \beta) \cos \left(4 \delta_{Q}\right) .
\end{aligned}
$$

The true value of $\delta_{Q}$ is an unknown function of wavelength that depends on the structure of the quarterwave plate used. Its apparent value can nevertheless be measured by use of a nonbirefringent sample; then the apparent birefringence is

$$
\tan ^{2}\left(\frac{\varphi_{\text {app }}}{2}\right)=\frac{\sin ^{2}\left(2 \delta_{Q}\right)}{1-\sin ^{2}\left(2 \delta_{Q}\right)} \approx \frac{4 \delta_{Q}{ }^{2}}{1-4 \delta_{Q}{ }^{2}} .
$$

We found experimentally that $\delta_{Q}=25 \mathrm{mrad}$; the value of $h$ can then be estimated from the apparent values of $\varphi_{\text {app }}$ and $\beta_{\text {app }}$. It is worth noting that $h$ does not depend on topography $\delta_{z}$. To calculate $h$ we need the sign of $\sin \left(\varphi_{\text {app }}\right) \times \sin \left(2 \beta_{\text {app }}\right)$. It can be found because, when $0 \leq \theta \leq \pi / 4$, one combination of the four signals $\Sigma_{A}{ }^{S}, \Sigma_{B}{ }^{S}, \Sigma_{A}{ }^{P}$, and $\Sigma_{B}{ }^{P}$ has the same sign:

$$
\begin{gathered}
\operatorname{sign}[\sin (\varphi) \sin (2 \beta)]=\operatorname{sign}\left[\Sigma_{A}^{(P)} \Sigma_{B}{ }_{B}^{(S)}-\Sigma_{A}{ }^{(S)} \Sigma_{B}{ }^{(P)}\right] \\
0 \leq \theta \leq \pi / 4
\end{gathered}
$$

The true value of $\varphi$ can then be estimated by

$$
\tan ^{2}\left(\frac{\varphi}{2}\right) \approx \frac{\cos ^{2}\left(\varphi_{\mathrm{app}} / 2\right)-h\left(\varphi_{\mathrm{app}}, \beta_{\mathrm{app}}, \delta_{Q}\right)}{\sin ^{2}\left(\varphi_{\mathrm{app}} / 2\right)+h\left(\varphi_{\mathrm{app}}, \beta_{\mathrm{app}}, \delta_{Q}\right)}
$$

The calibration plot of Fig. 3 was obtained with relation (15).

\section{Conclusion}

We have constructed a polarization-sensitive Linniktype interference microscope that works with a thermal light source. Our system uses full-field illumination with sinusoidal phase modulation and four integrating buckets. This system can produce en face $(x-y)$ tomographic images of the birefringence with a resolution of $1 \mu \mathrm{m}$ in the transverse direction and $1.5 \mu \mathrm{m}$ in the axial direction without scanning. Quantitative measurements can be obtained for birefringence from 0.1 to $3 \pi \mathrm{rad}$; these limits are fixed by the imperfections of the achromatic quarter-wave plates and the coherence length of the source. The maximum sensitivity of our setup, in terms of minimum detectable sample reflectance, was found to be $95 \mathrm{~dB}$ (84 dB for 1-s integration) for an individual image and $\sim 61 \mathrm{~dB}(\sim 50 \mathrm{~dB}$ for 1-s integration) for a birefringence measurement. We used our system as a dark-field microscope to detect and localize micrometer-sized defects inside a multilayer optical coating infrared mirror. Our primary interest was to gain a better understanding of the locations of scattering defects in multilayer optical coatings and therefore to determine the sources of contamination. Nevertheless, we are also currently investigating ways in which to use our microscope to study biological samples.

The authors thank Arnaud Dubois and Laurent Vabre for helpful discussions.

\section{References}

1. J. F. de Boer, T. E. Milner, M. J. C. van Gemert, and J. S. Nelson, "Two-dimensional birefringence imaging in biological tissue by polarization-sensitive optical coherence tomography," Opt. Lett. 22, 934-936 (1997).

2. J. F. de Boer, S. M. Srinivas, A. Malekafzali, Z. Chen, and J. S. Nelson, "Imaging thermally damaged tissue by polarization sensitive optical coherence tomography," Opt. Express 3, 212218 (1998), http://www.opticsexpress.org.

3. M. J. Everett, K. Schoenenberger, B. W. Colston, Jr., and L. B. Da Silva, "Birefringence characterization of biological tissue by use of optical coherence tomography," Opt. Lett. 23, 228-230 (1998).

4. K. Schoenenberger, B. W. Colston, Jr., D. J. Maitland, L. B. Da Silva, and M. J. Everett, "Mapping of birefringence and thermal damage in tissue by use of polarization-sensitive optical coherence tomography," Appl. Opt. 37, 6026-6036 (1998).

5. J. F. de Boer, T. E. Milnerand, and J. S. Nelson, "Determination of the depth-resolved Stokes parameters of light backscattered from turbid media by use of polarization-sensitive optical coherence tomography," Opt. Lett. 24, 300-302 (1999).

6. G. Yao and L. V. Wang, "Two-dimensional depth-resolved Mueller matrix characterization of biological tissue by optical coherence tomography," Opt. Lett. 24, 537-539 (1999).

7. C. E. Saxer, J. F. de Boer, B. H. Park, Y. Zhao, Z. Chen, and J. S. Nelson, "High-speed fiber-based polarization-sensitive optical coherence tomography of in vivo human skin," Opt. Lett. 25, 1355-1357 (2000).

8. C. K. Hitzenberger, E. Götzinger, M. Sticker, M. Pircher, and A. F. Fercher, "Measurement and imaging of birefringence and optic axis orientation by phase resolved polarization sensitive optical coherence tomography," Opt. Express 9, 780-790 (2001), http://www.opticsexpress.org.

9. J. E. Roth, J. A. Kozak, S. Yazdanfar, A. M. Rollins, and J. A. Izatt, "Simplified method for polarization-sensitive optical coherence tomography," Opt. Lett. 26, 1069-1071 (2001).

10. P. de Groot and L. Deck, "Surface profiling by analysis of white-light interferograms in the spatial frequency domain," J. Mod. Opt. 42, 389-401 (1995).

11. E. Beaurepaire, A. C. Boccara, M. Lebec, L. Blanchot, and H. Saint-Jalmes, "Full-field optical coherence microscopy," Opt. Lett. 23, 244-246 (1998).

12. A. Dubois, L. Vabre, A.-C. Boccara, and E. Beaurepaire, "Highresolution full-field optical coherence tomography with a Linnick microscope," Appl. Opt. 41, 805-812 (2002). 
13. J. Moreau and V. Loriette, "Full-field birefringence imaging by thermal-light polarization-sensitive optical coherence tomography. 1. Theory," Appl. Opt. 42, 3800-3810 (2003).

14. J. M. Mackowski, L. Pinard, L. Dognin, P. Ganau, B. Lagrange, C. Michel, and M. Morgue, "Different approaches to improve the wavefront of low-loss mirrors used in the VIRGO gravitational wave antenna," Appl. Surf. Sci. 151, 86-90 (1999).
15. J.-Y. Vinet, V. Brisson, S. Braccini, I. Ferrante, L. Pinard, F. Bondu, and E. Tournié, "Scattered light noise in gravitational wave interferometric detectors: a statistical approach," Phys. Rev. D 56, 6085-6095 (1997).

16. A. Dubois, "Phase-map measurements by interferometry with sinusoidal phase modulation and four integrating buckets," J. Opt. Soc. Am. A 18, 1972-1979 (2001). 